\title{
Phytochemical, GC-MS and FT-IR Analysis of Papaver somniferum L
}

\author{
S. Kumaravel ${ }^{1}$, P.Muthukumaran ${ }^{2 *}$, Nimia Thomas $^{3}$ \\ ${ }^{1}$ Professor, ${ }^{2}$ Senior Lecturer ${ }^{3}$ Student, ${ }^{1}$ Indian Institute of Food Processing Technology, Thanjavur, Tamil Nadu, ${ }^{2}$ DMI St. Eugene \\ University, 9 Miles, Great North Road, Chibombo, Zambia, ${ }^{3}$ Bishop Kurialacherry College, Kerala, India
}

Corresponding Author: P.Muthukumaran

Email: kumaran.bio14@gmail.com

\begin{abstract}
The present study was aimed to analysis of bioactive constituents of Papaver somniferum (Poppy seed). The ethanol extract of the seeds were subjected to Phytochemical Screening, Gas chromatography- mass spectroscopic (GC-MS) and Fourier transform infrared spectroscopy (FTIR) analysis. GC-MS analysis of the seeds was performed using a Scion 436- GC Bruker model nd Interpretation on mass spectrum GC-MS was conducted using the database of National Institute Standard and Technology (NIST) and IR spectrum was recorded in spectrophotometer (Shimadzu, IR Affinity1, Japan). Phytochemical screening for seeds extracts indicated the presence of various secondary metabolites like Alkaloid, Cardiac Glycosides, Flavonoid, Phytosterols and Terpenoids. GC-MS analysis of compounds with totally, Thirty Nine volatile compounds major chemical compounds were identified, such as 9-Octadecynoic acid(30.72\%), 9-Tetradecen1-ol, acetate, (E)- (24.02\%), 9,12-Octadecadienoic acid, methylester, (E,E)- (7.82\%), cis-9,10-Epoxyoctadecan-1-ol (7.43\%) and Undec10 -ynoic acid(4.36\%). FT-IR analysis of peak values with various functional compounds such as alcohols, phenols, carboxylic acids, aldehydes, amides, amino acids, anhydrides, esters, ketones, Unsaturated aliphatics, aromatics, unsaturated heterocycles, amines, Nitro compound, Alkanes, alkenes, sugars,Sulphur, phosphorus, and fluorine compounds. The present results concluded that the phytochemicals was observed in ethanol extract which revealed that the Papaver somniferum (Poppy seed) is potential use in different fields namely medical and pharmaceuticals and greatly valuable in medicinal practice for the treatment of several human aliments.
\end{abstract}

Keywords: GC-MS, FT-IR, Papaver somniferum L and NIST.

\section{Introduction}

Spices have been defined as plant substances from indigenous or exotic origin, aromatic or with strong taste, used to enhance the taste of foods (Pundir et al., 2010). Herbs and spices have been used for flavoring, food preservation, and/or medicinal commitments. Currently many ethnic cuisines are familiar for their reliance on "signature" herbs and spices. Several readings have endorsed the antimicrobial, antioxidant and pharmaceutical properties of spices and herbs to their phenolic compounds (Shan et al., 2005). Several studies have shown that spices are able to counteract oxidative stress in in vitro and in vivo systems (Ahmed et al., 2000). They extend the storage life of foods by preventing rancidity and oxidation of lipids (kelen and Tepe, 2008) or through bacteriostatic or bactericidal activity (Nazef et al., 2008) and they execute the antifungal activity (Kotzekidou et al., 2008). Spices and their extracts were had various therapeutic properties (Ayodele et al., 2009), they are affect digestion processes differently. Most of them stimulate the secretion of saliva.

Papaver somniferum L. belongs to the Papaveraceae family, and is commonly known as "Opium poppy." The plant is found wild in various parts of Europe, northern Africa, and western Asia (GRIN database 2009). It is traditionally used as an herbal medicine against coughing, bronchitis, sore throat, minor sleep problems, and possesses a sedative effect (Soulimani et al.,2001). Previous investigations on this plant have revealed its nutritional composition (Trichopoulou et al.,2000), content of alkaloids, (Kalav,and Sariyar, 2007) and ethnobotanical studies (Scherrer et al., 2005, Kultu, 2007) and (Cornara et al., 2009). Poppy seeds are used in traditional cuisine of several nations, mostly in confectionary and bakery food products such as fillings in cakes and desserts, or sprinkled on bread or rolls. (Erinç et al,. 2009). Moreover, they are a source of highly valuable oil, which is used not only for culinary purposes but also as an adjuvant for pharmaceutical and medical diagnostics, or as a component of cosmetic products and high-class oil-paints or varnishes (Krist et al., 2005).

GC-MS and FT-IR has played an important role in pharmaceutical analysis in recent years (Movasaghi et al., 2008), recently, spectroscopy has emerged as one of the major tools for biomedical claims and has made noteworthy progress in the field of clinical evaluation. Exploration has been accepted on a number of natural tissues using spectroscopic techniques, including FT-IR spectroscopy. GC-MS analysis is a breakthrough in analysis of phytoconstituents and structure elucidation of these compounds as they have a sensitivity of detecting compounds as low as 1ng (Liebler et al., 1996). The present study was carried out the bioactive compounds present in the Papaver somniferum L Spice in ethanol extract with the aid of GC-MS and FT-IR techniques, which may offer a perception in its use of out-dated medicine.

\section{Material and Methods \\ Extraction and Phytochemical Screening}

Papaver somniferum $\mathrm{L}$ were dried and powdered using a mixer blender to make fine powder. Then 2 grams of the powdered sample was added to $250 \mathrm{~mL}$ of solvent was eluted sequentially based on the polarity index of the solvents. Then the extracts were subjected for rotary evaporator and saved at fridge for future uses.

Preliminary qualitative analysis of phytochemical screening was performed with shade dried and powdered of 
the spice. The presence and absence of derivative compounds like alkaloids, carbohydrates, Phytosterols, flavonoids, phenolic, tannins, saponins, and terpenoids were confirmed by phytochemical screening using standard protocols (Harborne,1973).

\section{Preparation of Extracts for GC -MS}

$20 \mathrm{~g}$ of the powdered seeds of Papaversomniferum L. were soaked in $100 \mathrm{ml}$ of $95 \%$ methanol for $12 \mathrm{~h}$ and filtered through Whatmann filter paper No. 41 along with $2 \mathrm{~g}$ sodium sulfate to remove the deposits and traces of water in the remainder. The filtrate was then concentrated and the extract contained both polar and nonpolar phytocomponents of the plant material used. $2 \mu \mathrm{l}$ of this solution was used for GC/ MS analysis (Muthukumaran et al.,2017).

\section{GC Condition and Identification of Compounds}

The sample was examined through Gas Chromatography Mass Spectrometry/Mass Spectrometry Electron Ionization (GC-MS/EI) mode. The GC-MS/MS is a Scion 436- GC Bruker model coupled with a Triple quadruple mass spectrophotometer with fused silica capillary column BR5MS (5\% Diphenyl/95\% Dimethyl polysiloxane) and Length: 30m; Internal diameter: $0.25 \mathrm{~mm}$; Thickness: 0.25 $\mu \mathrm{m}$. Helium gas $(99.999 \%)$ was used as the carrier gas at a constant flow rate of $1 \mathrm{ml} / \mathrm{min}$ and an injection volume of 2 $\mu \mathrm{l}$ was working (split ratio of 10:1). The injector temperature $250^{\circ} \mathrm{C}$; ion-source temperature $280^{\circ} \mathrm{C}$.

The oven temperature was automated from $110^{\circ} \mathrm{C}$ (isothermal for $2 \mathrm{~min}$ ), with an increase of $10^{\circ} \mathrm{C} / \mathrm{min}$, to $200^{\circ} \mathrm{C}$, then $5^{\circ} \mathrm{C} / \mathrm{min}$ to $280^{\circ} \mathrm{C}$, windup with a $9 \mathrm{~min}$ isothermal at $280^{\circ} \mathrm{C}$ and total $\mathrm{GC}$ running time was $41 \mathrm{~min}$. This last escalation was to clean the column from any residues. The mass spectrometer was activated in the positive electron ionization (EI) mode with ionization energy of $70 \mathrm{eV}$. The solvent delay was 0-3.0 min. A scan intermission of 0.5 seconds and fragments from $\mathrm{m} / \mathrm{z} 50$ to $500 \mathrm{Da}$ was programmed. The inlet hotness was set at 280 ${ }^{\circ} \mathrm{C}$, source temperature $250{ }^{\circ} \mathrm{C}$. The relative fraction amount of each component was calculated by comparing its average peak area to the total areas. Software approved to handle mass spectra and chromatograms was MS Work station 8.

The NIST Version 2.0 library database of National Institute Standard and Technology (NIST) having more than 62,000 patterns was used for identifying the chemical components. The GC-MS/MS was performed by Food Safety and Quality Testing Laboratory, Indian Institute of Food Processing Technology, Thanjavur

\section{FTIR Spectroscopic Analysis}

Fourier transform infrared spectrophotometer (FTIR) is perhaps the most potent tools for identifying the types of chemical bonds (functional groups) present in compounds. Dry powders of altered solvent extracts of each plant material were used for FTIR analysis. 10mg of the dry extract powder was encapsulated in $100 \mathrm{mg}$ of $\mathrm{KBr}$ pellet, in orderto prepare translucent sample disc. The powdered sample of each plant specimen was loaded in FTIR
Spectroscope (Shimadzu, IR Affinity1, Japan), with a scan range from 400 to $4000 \mathrm{~cm}-1$ with a resolution of $4 \mathrm{~cm}-1$.

\section{Result and Discussion Phytochemical Analysis}

Spices have been supplementary to foods since ancient times as flavoring agent, also as food preservers and folk medicines. Spice is a natural compound that is extracted from the seeds, fruits, flowers or trunks (skin, roots, leaves) of several plants and add to food to provide taste, smell or flavor. Spices are staple dietary additives consumed all over the world (Farrell, 1990). Each spice has a unique aroma and flavor that derive from compounds known as phytochemicals or secondary metabolites. In the present study, the investigation of phytochemical screening was done by ethanol extract of Papaver somniferum L.The result revealed that the ethanolic extract of Papaver somniferum $\mathrm{L}$ recorded the presence of Alkaloid, Cardiac Glycosides, Flavonoid, Phytosterolsand Terpenoids whereas the CarbohydratesSaponins, Tannins were absent in the extract (Table 1).

These compounds involved in plants to protect against herbivorous insect vertebrates, fungi pathogens and parasites (Walker, 1994). For centuries the inherent value as well as potential; toxicity of phytochemicals to human health has been recognized (Charaka, 1994). Spices are used as the substances that increase the taste and variation of food (Bulduk, 2004). The spices, herbs, plant extract and their phytoconstituents have been informed for antiinflammatory, antidiarrheal, antimicrobial, antioxidant and insecticidal activities (Chouhan and Singh, 2011)

Table1: Phytochemical screening of Papaver somniferum L

\begin{tabular}{|c|c|}
\hline Phytochemical & Poppy seed \\
\hline Alkaloids & + \\
\hline Carbohydrate & - \\
\hline Cardiac Glycosides & + \\
\hline Flavonoids, & + \\
\hline Phytosterols & + \\
\hline Saponins & - \\
\hline Tannins & - \\
\hline Terpenoids, & + \\
\hline
\end{tabular}

+ Present - Absent

\section{GC MS Analysis}

The compounds present in the ethanolic extract of Papaver somniferum L, were identified by GC-MS analysis (Fig. 1). Thirty Nine volatile compounds from ethanolic extract of Papaver somniferum $\mathrm{L}$ were separated and identified by GCMS. The components identified, molecular formulae, molecular weight and the time of elution with peak area were delivered in Table 2.

The GC-MS analyses of Papaver somniferum L established the identification of 39 volatile compounds in the ethanolic extract. The composition are as follows: 9Octadecynoic acid (30.72\%), 9-Tetradecen-1-ol, acetate, (E)- (24.02\%), 9,12-Octadecadienoic acid, methyl ester, 
(E,E)- (7.82\%), cis-9,10-Epoxyoctadecan-1-ol (7.43\%) and Undec-10-ynoic acid (4.36\%). The chemical group classifications are as follows: Monoterpenes (1.33\%), Aromatic (0.47\%), Amino acid (1.42), Fatty acid (51.03\%), Acetate (24.31\%), Nitrogen compounds (0.14\%), Alcohol
(0.73\%), Aldehyde (0.33\%), Alkanes (1.22\%), Alkenes (1.07), Esters (0.94\%), Epoxy compounds (2.23\%), naphthalene $(0.71 \%)$ and ketones $(0.75 \%)$.

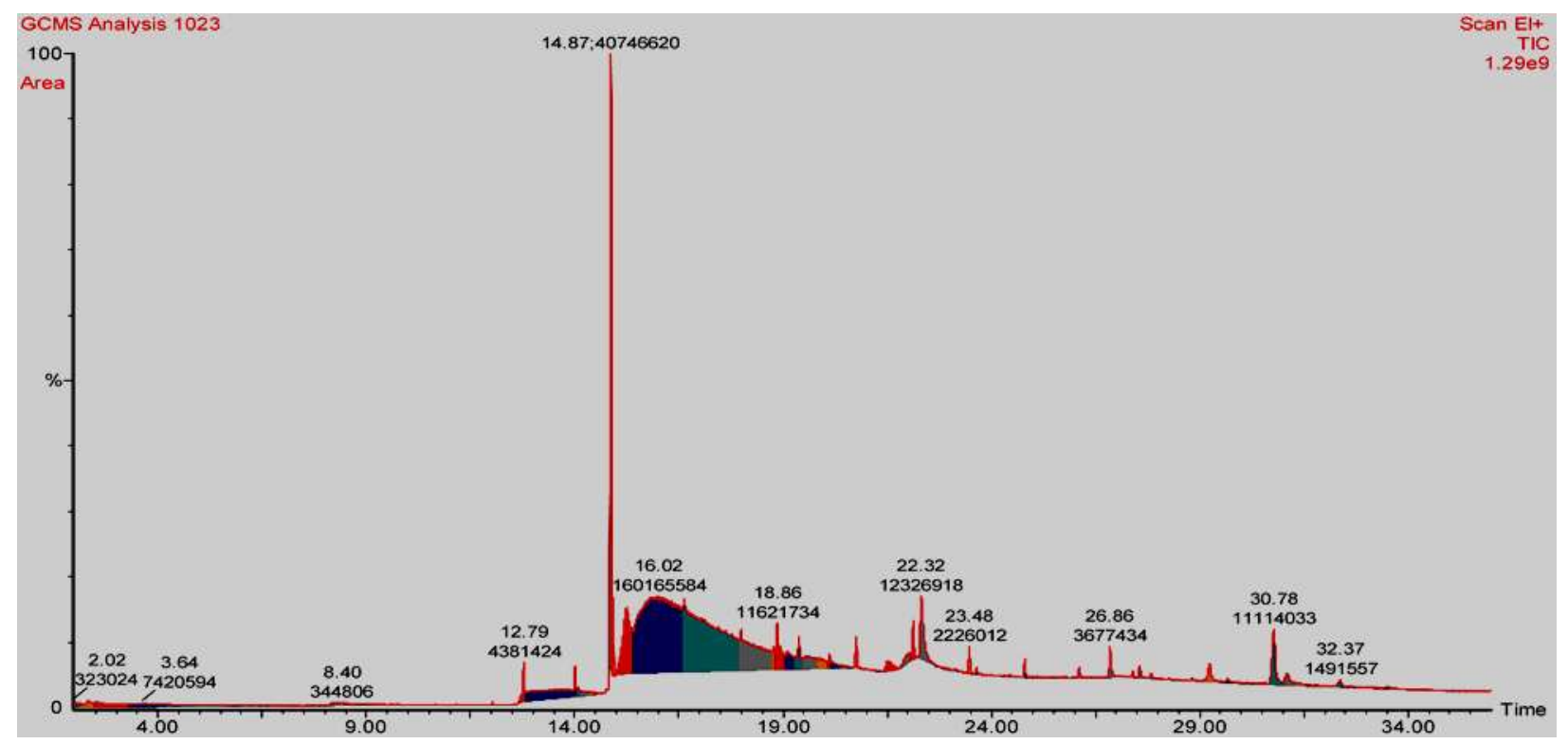

Fig. 1: Peak area percentage of (GC-MS) Gas column mass spectrometry in Papaver somniferum L

Table 2: GC-MS analysis revealed the presence of bioactive compounds in the Papaversomniferum L (Poppy seeds).

\begin{tabular}{|c|c|c|}
\hline S. No & Identified Compound Details & Activity \\
\hline 1 & $\begin{array}{c}\text { á-Pinene(RT-2.06), Molecular } \\
\text { Formula- } \mathrm{C}_{10} \mathrm{H}_{16} \text {, MW -136, Peak } \\
\text { Area\% -0.12, } \\
\text { CompoundNatureMonoterpene }\end{array}$ & $\begin{array}{l}\text { Anti-inflammatory, Sedative, Anticancer, Antitumor, } \\
\text { Antibacterial, Antiflu, Nematicide, Insecticide, Pesticide, } \\
\text { Herbicide. Flavor, Immunomodulator, Fungistat, Antiobesity, } \\
\text { Detoxicant, Chemo preventive, Expectorant, Photo sensitizer }\end{array}$ \\
\hline 2 & $\begin{array}{c}\text { Benzene, 1-methyl-3-(1-methylethyl) } \\
\text { (RT-2.33),Molecular Formula- } \\
\mathrm{C}_{10} \mathrm{H}_{14} \text {, MW -134, Peak Area\% - } \\
0.47 \text {, CompoundNature-Aromatic } \\
\text { compound }\end{array}$ & No activity reported \\
\hline 3 & $\begin{array}{l}\text { 1,4-Cyclohexadiene, 1-methyl-4-(1- } \\
\text { methylethyl)-,(RT-2.53),Molecular } \\
\text { Formula- } \mathrm{C}_{10} \mathrm{H}_{16} \text {, MW -136, Peak } \\
\text { Area\% -1.21, CompoundNature- } \\
\text { Monoterpene }\end{array}$ & $\begin{array}{l}\text { Anti-inflammatory, Sedative, Anticancer, Antitumor, } \\
\text { Antibacterial, Antiflu, Nematicide, Insecticide, Pesticide } \\
\text { Herbicide, Flavor, Immunomodulator, Fungistat, Antiobesity, } \\
\text { Detoxicant, Chemo preventive, Expectorant, Photo sensitizer }\end{array}$ \\
\hline 4 & $\begin{array}{l}\text { Butanoic acid, 4-(dimethylamino)-3- } \\
\text { hydroxy,(RT-3.64),Molecular } \\
\text { Formula- } \mathrm{C}_{6} \mathrm{H}_{13} \mathrm{NO}_{3} \text {, MW -147, } \\
\text { Peak Area\% -1.42, CompoundNature- } \\
\text { Amino compound }\end{array}$ & Antimicrobial \\
\hline 5 & $\begin{array}{l}\text { 3-Ethylheptanoic acid,(RT- } \\
\text { 6.01), Molecular Formula- } \mathrm{C}_{9} \mathrm{H}_{18} \mathrm{O}_{2} \text {, } \\
\text { MW -158, Peak Area\% -0.04, } \\
\text { CompoundNature- Fatty acid } \\
\text { compound }\end{array}$ & No activity reported \\
\hline 6 & $\begin{array}{l}\text { 1,3-Propanediol, 2-(hydroxymethyl)- } \\
\text { 2-nitro-,(RT-8.40),Molecular } \\
\text { Formula- } \mathrm{C}_{4} \mathrm{H}_{9} \mathrm{NO}_{5}, \mathrm{MW}-151, \text { Peak }\end{array}$ & Antimicrobial \\
\hline
\end{tabular}




\begin{tabular}{|c|c|c|}
\hline & $\begin{array}{l}\text { Area\% - } 0.07 \text {, CompoundNature- } \\
\text { Nitrogen compound }\end{array}$ & \\
\hline 7 & $\begin{array}{l}\text { 1,3-Propanediol, 2-(hydroxymethyl)- } \\
\text { 2-nitro-,(RT-8.40),Molecular } \\
\text { Formula- C4H9NO5, MW -151, Peak } \\
\text { Area\% -0.07, CompoundNature- } \\
\text { Nitrogen compound } \\
\end{array}$ & Antimicrobial \\
\hline 8 & $\begin{array}{l}\text { Cyclopentaneundecanoic acid, methyl } \\
\text { ester-,(RT-12.04), Molecular } \\
\text { Formula- C17H32O2, MW -268, Peak } \\
\text { Area\% -0.05, CompoundNature- Fatty } \\
\text { acid ester } \\
\end{array}$ & No activity reported \\
\hline 9 & $\begin{array}{l}\text { Tetradecanoic acid, ethyl ester-,(RT- } \\
\text { 12.79),Molecular Formula- } \\
\mathrm{C}_{16} \mathrm{H}_{32} \mathrm{O}_{2} \text {, MW -256, Peak Area\% - } \\
\text { 0.84, CompoundNature-Myristic acid } \\
\text { ester }\end{array}$ & $\begin{array}{c}\text { Nematicide,Antioxidant,Cosmetic } \\
\text { Cancer preventive,Hypercholestrolemic } \\
\text { Lubricant }\end{array}$ \\
\hline 10 & $\begin{array}{c}\text { Undec-10-ynoic acid-,(RT- } \\
\text { 14.08),Molecular Formula- } \\
\mathrm{C}_{11} \mathrm{H}_{18} \mathrm{O}_{2}, \mathrm{MW}-182 \text {, Peak Area\% - } \\
\text { 4.36, CompoundNature- Unsaturated } \\
\text { fatty acid } \\
\end{array}$ & No activity reported \\
\hline 11 & $\begin{array}{c}\text { n-Hexadecanoic acid-, }(\mathrm{RT}-14.10), \\
\text { Molecular Formula- } \mathrm{C}_{16} \mathrm{H}_{32} \mathrm{O}_{2}, \mathrm{MW} \\
-256, \text { Peak Area\% }-0.22, \\
\text { CompoundNature-Palmitic acid } \\
\end{array}$ & $\begin{array}{l}\text { Antioxidant HypocholesterolemicNematicide Pesticide, Anti } \\
\text { androgenic FlavorHemolytic 5-Alpha reductase inhibitor }\end{array}$ \\
\hline 12 & $\begin{array}{c}\text { Undecanoic acid-,(RT-14.17), } \\
\text { Molecular Formula- } \mathrm{C}_{11} \mathrm{H}_{22} \mathrm{O}_{2}, \mathrm{MW} \\
\quad-186, \text { Peak Area\% }-0.38, \\
\text { CompoundNature- Saturated fatty acid }\end{array}$ & No activity reported \\
\hline 13 & $\begin{array}{l}\text { 9,12-Octadecadienoic acid, methyl } \\
\text { ester, (E,E),(RT-14.87),Molecular } \\
\text { Formula- } \mathrm{C}_{19} \mathrm{H}_{34} \mathrm{O}_{2}, \mathrm{MW}-294, \text { Peak } \\
\text { Area\% -7.82, CompoundNature- } \\
\text { Linoleic acid ester }\end{array}$ & $\begin{array}{l}\text { Hypocholesterolemic,Nematicide,Antiarthritic,Hepatoprotective, } \\
\text { Anti androgenic, 5-Alpha reductase inhibitor } \\
\text { Antihistaminic,Anticoronary, InsectifugeAntieczemic,Antiacne }\end{array}$ \\
\hline 14 & $\begin{array}{l}\text { 9,12-Octadecadienoic acid (Z,Z)-(RT- } \\
\text { 14.93),Molecular Formula- } \\
\mathrm{C}_{18} \mathrm{H}_{32} \mathrm{O}_{2}, \mathrm{MW} \text {-280, Peak Area\% - } \\
0.78, \text { CompoundNature- Linoleic acid } \\
\text { ester } \\
\end{array}$ & $\begin{array}{l}\text { HypocholesterolemicNematicideAntiarthriticHepatoprotective } \\
\text { Anti androgenic 5-Alpha reductase inhibitor Antihistaminic } \\
\text { AnticoronaryInsectifugeAntieczemicAntiacne }\end{array}$ \\
\hline 15 & $\begin{array}{l}\text { 11,14-Eicosadienoic acid, methyl } \\
\text { ester(RT-15.26),Molecular Formula- } \\
\mathrm{C}_{21} \mathrm{H}_{38} \mathrm{O}_{2}, \mathrm{MW}-322 \text {, Peak Area\% - } \\
\text { 5.81, CompoundNature- Unsaturated } \\
\text { fatty acid ester }\end{array}$ & Cardio protective \\
\hline 16 & $\begin{array}{c}\text { 9-Octadecynoic acid(RT- } \\
\text { 16.02),Molecular Formula- } \\
\mathrm{C}_{18} \mathrm{H}_{32} \mathrm{O}_{2} \text {, MW -280, Peak Area\% - } \\
\text { 30.72, CompoundNature- Unsaturated } \\
\text { fatty acid ester } \\
\end{array}$ & No activity reported \\
\hline & $\begin{array}{c}\text { 9-Tetradecen-1-ol, acetate, (E)- (RT- } \\
\text { 16.63),Molecular Formula- } \\
\mathrm{C}_{16} \mathrm{H}_{30} \mathrm{O}_{2} \text {, MW -254, Peak Area\% - } \\
\text { 24.02, CompoundNature- Acetate } \\
\text { compound }\end{array}$ & No activity reported \\
\hline
\end{tabular}




\begin{tabular}{|c|c|c|}
\hline 18 & $\begin{array}{c}\text { cis-9,10-Epoxyoctadecan-1-ol(RT- } \\
\text { 18.00)Molecular Formula- } \\
\mathrm{C}_{18} \mathrm{H}_{36} \mathrm{O}_{2} \text {, MW _284, Peak Area\% - } \\
\text { 7.43, CompoundNature- Alcoholic } \\
\text { compound } \\
\end{array}$ & Antimicrobial \\
\hline 19 & $\begin{array}{c}\text { 1,2-15,16-Diepoxyhexadecane(RT- } \\
18.86) \mathrm{C}_{16} \mathrm{H}_{30} \mathrm{O}_{2}, \mathrm{MW}-254, \text { Peak } \\
\text { Area\% -2.23, CompoundNature- } \\
\text { Epoxy compound }\end{array}$ & No activity reported \\
\hline 20 & $\begin{array}{c}\text { Dodecane, 2,6,10-trimethyl,(RT- } \\
\text { 19.39)Molecular Formula- } \mathrm{C}_{15} \mathrm{H}_{32} \text {, } \\
\text { MW -212, Peak Area\% -1.01, } \\
\text { CompoundNature- Alkane compound }\end{array}$ & No activity reported \\
\hline 21 & $\begin{array}{l}\text { 9,12-Octadecadienal-(RT- } \\
\text { 20.11)Molecular Formula- } \mathrm{C}_{15} \mathrm{H}_{32} \text {, } \\
\text { MW -264, Peak Area\% -0.33, } \\
\text { CompoundNature- Aldehyde } \\
\text { compound }\end{array}$ & $\begin{array}{c}\text { Antimicrobial } \\
\text { Anti-inflammatory }\end{array}$ \\
\hline 22 & $\begin{array}{c}\text { Methoxyacetic acid, 4-tetradecyl } \\
\text { ester-(RT-20.76)Molecular Formula- } \\
\mathrm{C}_{17} \mathrm{H}_{34} \mathrm{O}_{3} \text {, MW -286, Peak Area\% - } \\
\text { 0.51, CompoundNature Ester } \\
\text { compound }\end{array}$ & No activity reported \\
\hline 23 & $\begin{array}{c}\text { E,E-1,9,17-Docasatriene,(RT- } \\
\text { 21.51)Molecular Formula- } \mathrm{C}_{22} \mathrm{H}_{40} \text {, } \\
\text { MW -304, Peak Area\% -0.70, } \\
\text { CompoundNature Alkene compound }\end{array}$ & No activity reported \\
\hline 24 & $\begin{array}{c}\text { cisZ-11,12-Epoxytetradecan-1-ol,(RT- } \\
\text { 22.13)Molecular Formula- } \\
\mathrm{C}_{14} \mathrm{H}_{28} \mathrm{O}_{2} \text {, MW- } 228 \text {, Peak Area\% - } \\
\text { 0.68, CompoundNature-Alcoholic } \\
\text { compound } \\
\end{array}$ & Antimicrobial \\
\hline 25 & $\begin{array}{l}\text { (Z)6,(Z)9-Pentadecadien-1-ol,(RT- } \\
\text { 22.32)Molecular Formula- } \mathrm{C}_{15} \mathrm{H}_{28} \mathrm{O} \text {, } \\
\text { MW -224, Peak Area\% -2.36, } \\
\text { CompoundNature Alcoholic } \\
\text { compound }\end{array}$ & Antimicrobial \\
\hline 26 & $\begin{array}{l}\text { Methoxyacetic acid, 3-tetradecyl } \\
\text { ester,(RT-23.48)Molecular Formula- } \\
\mathrm{C}_{17} \mathrm{H}_{34} \mathrm{O}_{3} \text {, MW -286, Peak Area\% - } \\
\text { 0.43, CompoundNature- Ester } \\
\text { compound }\end{array}$ & No activity reported \\
\hline 27 & $\begin{array}{c}\text { 1,E-11,Z-13-Octadecatriene, }(\mathrm{RT}- \\
\text { 23.65)Molecular Formula- } \mathrm{C}_{18} \mathrm{H}_{32} \text {, } \\
\text { MW -248, Peak Area\% - } 0.13 \text {, } \\
\text { CompoundNature-Alkene compound }\end{array}$ & No activity reported \\
\hline 28 & $\begin{array}{l}\text { trans-2-Undecen-1-ol,(RT- } \\
\text { 24.81)Molecular Formula- } \mathrm{C}_{11} \mathrm{H}_{22} \mathrm{O} \text {, } \\
\text { MW -170, Peak Area\% -0.31, } \\
\text { CompoundNature- Alcoholic } \\
\text { compound }\end{array}$ & Antimicrobial \\
\hline 29 & $\begin{array}{l}\text { E-2-Tetradecen-1-ol,(RT- } \\
\text { 26.11)Molecular Formula- } \mathrm{C}_{14} \mathrm{H}_{28} \mathrm{O} \text {, } \\
\text { MW -212, Peak Area\% -0.21, }\end{array}$ & Antimicrobial \\
\hline
\end{tabular}




\begin{tabular}{|c|c|c|}
\hline & $\begin{array}{l}\text { CompoundNature- Alcoholic } \\
\text { compound }\end{array}$ & \\
\hline 30 & $\begin{array}{l}\text { Naphthalene, decahydro-2,2-dimethyl- } \\
\text {,(RT-26.86)Molecular Formula- } \\
\mathrm{C}_{12} \mathrm{H}_{22} \text {, MW -166, Peak Area\% - } \\
\text { 0.71, CompoundNature- Naphthalene } \\
\text { compound } \\
\end{array}$ & No activity reported \\
\hline 31 & $\begin{array}{c}\text { 2-Hydroxy-(Z)9-pentadecenyl } \\
\text { propanoate-,(RT-27.56)Molecular } \\
\text { Formula- } \mathrm{C}_{18} \mathrm{H}_{34} \mathrm{O}_{3} \text {, MW _-298, Peak } \\
\text { Area\% }-0.29, \text { CompoundNature- } \\
\text { Hydroxy compound } \\
\end{array}$ & No activity reported \\
\hline 32 & $\begin{array}{l}\text { 13-Oxabicyclo[10.1.0]tridecane-,(RT- } \\
\text { 27.84)Molecular Formula- } \mathrm{C}_{12} \mathrm{H}_{22} \mathrm{O} \text {, } \\
\text { MW -182, Peak Area\% }-0.13 \text {, } \\
\text { CompoundNature- Alkane compound }\end{array}$ & No activity reported \\
\hline 33 & $\begin{array}{c}\text { E,E-1,9,17-Docasatriene-, (RT- } \\
\text { 28.83)Molecular Formula- } \mathrm{C}_{22} \mathrm{H}_{40} \text {, } \\
\text { MW -304, Peak Area\% -0.07, } \\
\text { CompoundNature- Alkene compound }\end{array}$ & No activity reported \\
\hline 34 & $\begin{array}{c}\text { Dodeca-1,6-dien-12-ol, 6,10- } \\
\text { dimethyl--,(RT-29.24)Molecular } \\
\text { Formula- } \mathrm{C}_{14} \mathrm{H}_{26} \mathrm{O}, \mathrm{MW}-210, \text { Peak } \\
\text { Area\% -0.73, CompoundNature- } \\
\text { Unsaturated alcoholic compound }\end{array}$ & No activity reported \\
\hline 35 & $\begin{array}{c}\text { Z,Z,Z-4,6,9-Nonadecatriene-,(RT- } \\
\text { 29.69)Molecular Formula- } \mathrm{C}_{19 \mathrm{H}} 34 \text {, } \\
\text { MW -262, Peak Area\% -0.17, } \\
\text { CompoundNature- Alkene compound }\end{array}$ & No activity reported \\
\hline 36 & $\begin{array}{c}\text { 5à-Androstan-16-one, cyclic ethylene } \\
\text { mercaptole-,(RT-30.78)Molecular } \\
\text { Formula- } \mathrm{C}_{21} \mathrm{H}_{34} \mathrm{~S}_{2}, \mathrm{MW}-350 \text {, Peak } \\
\text { Area\% -2.13, CompoundNature- } \\
\text { Steroid }\end{array}$ & $\begin{array}{l}\text { Antimicrobial Anti-inflammatory Anticancer Diuretic } \\
\text { Antiarthritic Antiasthma }\end{array}$ \\
\hline 37 & $\begin{array}{c}\text { Oxacycloheptadec-8-en-2-one-,(RT- } \\
31.11) \text { Molecular Formula- } \\
\mathrm{C}_{16} \mathrm{H}_{28} \mathrm{O}_{2} \text {, MW -252, Peak Area\% - } \\
\text { 0.75, CompoundNature- Ketone } \\
\text { compound } \\
\end{array}$ & No activity reported \\
\hline 38 & $\begin{array}{l}\text { cis-7,cis-11-Hexadecadien-1-yl } \\
\text { acetate-, (RT-32.37)Molecular } \\
\text { Formula- } \mathrm{C}_{18} \mathrm{H}_{32} \mathrm{O}_{2}, \mathrm{MW}-280 \text {, Peak } \\
\text { Area\% -0.29, CompoundNature- } \\
\text { Acetate compound }\end{array}$ & No activity reported \\
\hline 39 & $\begin{array}{l}\text { 12-Methyl-E,E-2,13-octadecadien-1- } \\
\text { ol-,(RT-33.51)Molecular Formula- } \\
\mathrm{C}_{19} \mathrm{H}_{36} \mathrm{O}, \mathrm{MW} \text {-280, Peak Area\% - } \\
\text { 0.08, CompoundNature- Unsaturated } \\
\text { alcoholic compound }\end{array}$ & No activity reported \\
\hline
\end{tabular}

The functional therapeutic activity of the poppy seed compounds were identified through Dr. Duke's Phytochemical Database. The fatty acids which constitute $51.03 \%$ possess antioxidant activity and also the anti-inflammatory activity. Compounds namely, á-Pinene, 1,4-Cyclohexadiene, 1-methyl-4-(1-methylethyl)-, n-Hexadecanoic acid are having insecticide activity and proven for pesticide activity. Flavors compounds like ketones, aldehydes and alcohols were enriched in poppy seed. The present study indicates that poppy seed is a good natural source of sterols. In addition, the findings in this study are 
important for the nutrition sciences, because fatty acids and phytosterols, in particular, seem to have considerable effects on health.

\section{FTIR Analysis of Papaver somniferum}

The FT-IR spectrum was used to find the functional groups of the active components present in extract based on the peaks values in the region of IR radiation. Once the extract was passed into the FT-IR, the functional groups of the components were separated based on its peaks ratio.

The ethanolic extract of Papaver somniferum L showed characteristic absorption bands at $3285.85 \mathrm{~cm}-1$ for O-H stretching vibration presence of alcohols, phenols, $2925.05 \mathrm{~cm}-1(\mathrm{O}-\mathrm{H}$ stretching vibration presence of carboxylic acids), $2855.04 \mathrm{~cm}-1$ (CHO Aldehydes (Fermi doublet), $1744.18 \mathrm{~cm} \mathrm{-1(C=O} \mathrm{Acid} \mathrm{halides,} \mathrm{aldehydes,} \mathrm{amides,} \mathrm{amino} \mathrm{acids,}$ anhydrides, carboxylic acids, esters, ketones, lactams, lactones, quinines), $1637.03 \mathrm{~cm}-1(\mathrm{C}=\mathrm{C}, \mathrm{C}=\mathrm{N}, \mathrm{NH}$ Unsaturated aliphatics, aromatics, unsaturated heterocycles, amides, amines, amino acids), $1545.82 \mathrm{~cm}-1$ (NO2 Nitro compound $\mathrm{CH} 3$ and $\mathrm{CH} 2$ Alkanes, alkenes), $1454.4 \mathrm{~cm}-1(\mathrm{C}-\mathrm{H}$ bend stretching vibration presence of alkenes), $1313.89 \mathrm{~cm}-1(\mathrm{~N}-\mathrm{O}$ stretching vibration presence of nitro compounds), $1235.3 \mathrm{~cm}-1(\mathrm{C}-\mathrm{O}-\mathrm{C}$ and $\mathrm{C}-\mathrm{OH}$ Ethers, alcohols, sugars $\mathrm{S}=\mathrm{O}, \mathrm{P}=\mathrm{O}, \mathrm{C}-\mathrm{F}$ Sulphur, phosphorus, and fluorine compounds) and $1049.35 \mathrm{~cm} \mathrm{-1}$ for Si-O and P-O Organosilicon and phosphorus compounds (Fig. 2 \& Table 3)

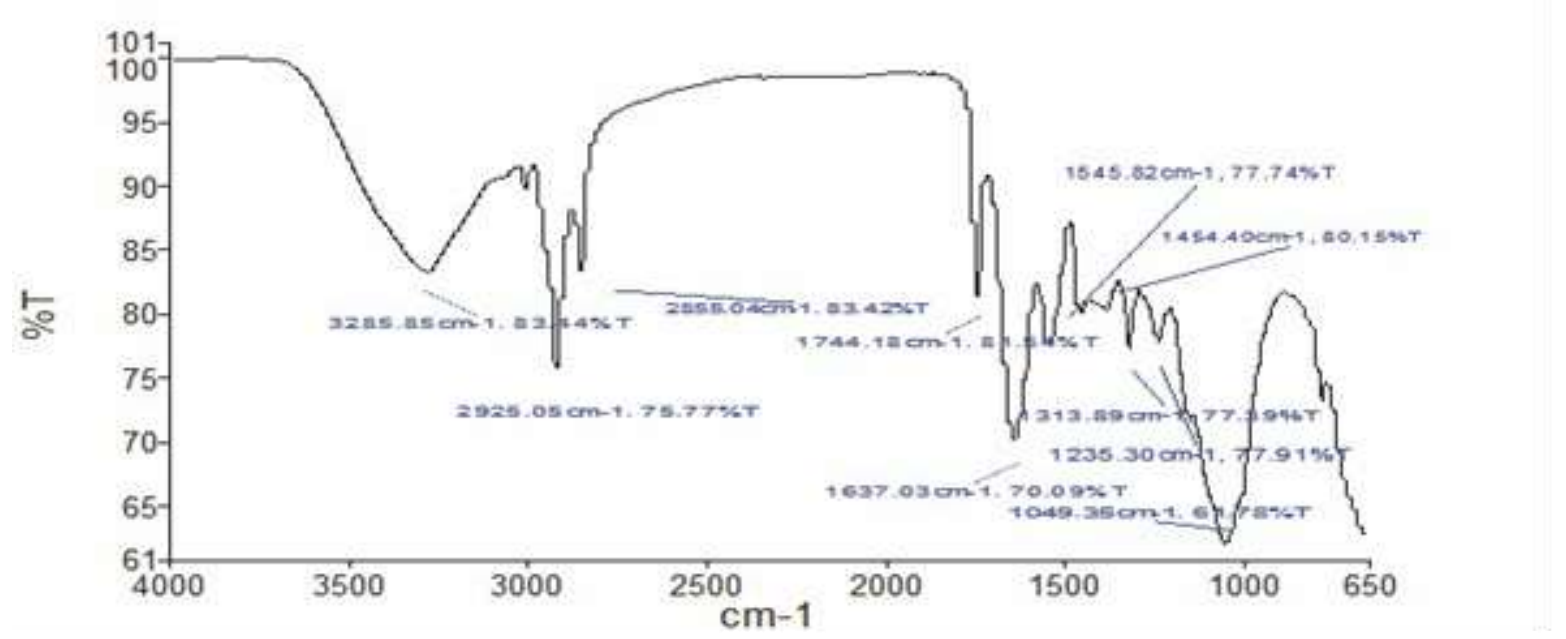

Fig. 2: FTIR- Spectrum wave numbers of Papaver somniferum $L$

Table 3: FTIR Analysis of Papaver somniferum L

\begin{tabular}{|c|c|c|c|}
\hline S. No & Peak values & $\begin{array}{c}\text { Frequency } \\
\operatorname{ranges}\left(\mathrm{cm}^{-1}\right)\end{array}$ & Functional groups and Possible compounds \\
\hline 1 & 3285.85 & $3500-3200$ & O-H stretchingvibrationpresenceof alcohols, phenols \\
\hline 2 & 2925.05 & $3300-2500$ & $\mathrm{O}-\mathrm{H}$ stretchingvibrationpresenceof carboxylicacids \\
\hline 3 & 2855.04 & $2800-2600$ & -CHOAldehydes (Fermi doublet) \\
\hline 4 & 1744.18 & $1870-1650$ & $\begin{array}{c}\mathrm{C}=\mathrm{O} \text { Acid halides, aldehydes, amides, amino acids, anhydrides, carboxylic } \\
\text { acids, esters, ketones, lactams, lactones, quinines }\end{array}$ \\
\hline 5 & 1637.03 & $1650-1550$ & $\begin{array}{c}\mathrm{C}=\mathrm{C}, \mathrm{C}=\mathrm{N}, \mathrm{NH} \text { Unsaturated aliphatics, aromatics, unsaturated heterocycles, } \\
\text { amides, amines, aminoacids }\end{array}$ \\
\hline 6 & 1545.82 & $1550-1300$ & NO2NitrocompoundCH3andCH2Alkanes, alkenes, etc \\
\hline 7 & 1454.4 & $1470-1450$ & $\mathrm{C}-\mathrm{H}$ bend stretchingvibrationpresenceof alkenes \\
\hline 8 & 1313.89 & $1400-1290$ & $\mathrm{~N}$-Ostretchingvibrationpresenceofnitro compounds \\
\hline 9 & 1235.3 & $1300-1000$ & $\begin{array}{l}\text { C-O-C and C-OH Ethers, alcohols,sugars } \mathrm{S}=\mathrm{O}, \mathrm{P}=\mathrm{O}, \mathrm{C}-\mathrm{F} \text { Sulphur, } \\
\text { phosphorus, and fluorine compounds }\end{array}$ \\
\hline 10 & 1049.35 & $1100-800$ & Si-O and P-O Organ silicon andphosphorus compounds \\
\hline
\end{tabular}

\section{Conclusion}

The presence of naturally active compounds also contributes to its healthy value and thus proved to be potential sources of useful foods. Additionally, isolation, purification and characterization of the phytochemicals will make remarkable studies. The result of this study would lead to discovery of some compounds which are very useful for the manufacturing of new drugs. This primary information will simplify in leading further studies on discovery of bioactive 
ingredients, resolve of their efficacy by in vivo studies and demonstration of their safety and efficacy in clinical trials.

\section{Acknowledgement}

The authors are thankful to Director, Indian Institute of Food Processing Technology, Thanjavur, Tamil Nadu, India for providing all the facilities to conduct this work.

\section{Conflict of Interest: None.}

\section{References}

1. Ahmed RS, Seth V, Banerjee BD. Influence of dietary ginger (Zingiber officinales Rosc) on oxidative stress induced by malathion in rats. Food ChemToxicol. 2000;38:443-50.

2. Ayodele S.M., Llondu E.M. and Onwubolu N.C. Antifungal properties of some locally used spices In Nigeria against some rot fungi. Afr J Plant Sci 2009;36:139-41

3. Bulduk S. (2004). Food Technology. 2nd edition, Detay Publishing, Ankara, Turkey.

4. Charaka, Charak Samhita and Sutra Sthan. 3rd 4. Edition. Chaukamba Surbharati Prakashan,Varanasi. 1994.

5. Chouhan H.S., Singh S.K.A. Review of plants of genus Leucas. J Pharmacognosy Phytotherapy 2001;3(3):13-26

6. Cornara, L. La Rocca, A. Marsili, S. Mariotti, M.G.(2009). "Traditional uses of plants in the Eastern Riviera (Liguria, Italy)," J Ethnopharmacol 125:16-30

7. Erinç, D. D. Tekin, A. Özcan, M. M.(2009). Determination of fatty acid, tocopherol and phytosterol contents of the oils of various poppy (Papaversomniferum L.) seeds. Grasas Aceites 60:375-81.

8. Farrell KT. (1990).Spices, Condiments, and Seasonings. $2^{\text {nd }}$ New York: Van Nostrand Reinhold.

9. GRIN database. Germplasm Resources Information Network(GRIN) (2009).National Germplasm Resources Laboratory, Beltsville, Maryland,.Retrieved from http:/www.arsgrin.gov/cgibin/ html/tax.

10. Harborne, J. B. (1973)"Phenolic compounds." Phytochemical methods. Pringer Netherlands 33-88.

11. Kalav,Y.N. and Sarıyar,G. "Alkaloids from Turkish Papaverrhoeas," Planta Medica 1989;5:488.

12. Kelen M. and Tepe B. Chemical composition, antioxidant and antimicrobial properties of the essential oils of three Salvia species from Turkish flora. Bioresour. Technol 2008;99:40964104

13. Kotzekidou P., Giannakidis P. and Boulamatsis A. Antimicrobial activity of some plant extracts and essential oils against food-borne pathogens in vitro and on the fate of inoculated pathogens in chocolate. LWT 2008;41:119-27

14. Krist, S. Stuebinger, G. Unterweger, H. Bandion, F. Buchbauer, G. Analysis of volatile compounds and triglycerides of seed oils extracted from different poppy varieties (Papaversomniferum L.). J Agricultural Food Chemistry 2005;53:8310-16.

15. Kultur,S.(2007). "Medicinal plants used in Kirklareli Province (Turkey)," J Ethnopharmacol 111:341-64.

16. Liebler D.C., Burr J.A., Philips L., Ham A.J.L.(1996). Gas chromatography - mass spectrometry analysis of vitamin E and its oxidation products. Anal Biochem 236:27-34.

17. Movasaghi Z., Rehman S., Rehman I.U. (2008).Fourier transform infrared spectroscopy of biological tissues. Appl Spec Rev 43:134-79.

18. Muthukumaran, P., Suresh Kumar and Karthikeyen, R. Phytochemical Screening, GC-MS, FT-IR Analysis of Methanolic Extract of Curcuma caesiaRoxb (Black Turmeric). Pharmacog J 2017;9(6):952-956.

19. Nazef L., Belguesmia Y., Tani A., Prevost H. and Drider D. Identification of lactic acid bacteria from poultry feces: Evidence on anti- Campylobacter and anti-Listeria activities. Poultry Sci 2008;872:329- 34.

20. Oancea A., Roată G., Popescu S. Phytochemical screening of the bioactive compounds in the most widespread medicinal plants from calarasi-silistra cross-border area. Bulletin of the Transilvania University of Braşov. 2013;135-142.

21. Pundir RK, Jain P, Sharma C. Antimicrobial activity of ethanolic extracts of Syzygiumaromaticum and Allium sativum against food associated bacteria and fungi. Ethnobot Leaflets 2010;14:344-60.

22. Scherrer, A.M. Motti,R and Weckerle, C.S. "Traditional plant use in the areas of Monte Vesole and Ascea, Cilento National Park (Campania, Southern Italy)," J Ethnopharmacol 2005;97:129-43.

23. Shan B, Cai YZ, Sun M, Corke H. Antioxidant capacity of 26 spice extracts and characterisation of their phenolic constituents. J Agric Food Chem. 2005;53(20):7749-59.

24. Soulimani, R. Younos,C. Jarmouni-Idrissi, S. Bousta,D Khalouki, F. Laila, A. "Behavioral and pharmacotoxicological study of Papaverrhoeas L. in mice," J Ethnopharmacol 2001;74:265-74,

25. Trichopoulou, A. Vasilopoulou, E. Hollman, P. Chamalides, C.H. Foufa, E. Kaloudis, T. R. Kromhout, D. Miskaki, P. H. Etrochilou, I. Poulima, E. Stafilakis, K. and Theophilou, D. (2000). "Nutritional composition and flavonoid content of edible wild greens and green pies: A potentialrich source of antioxidant nutritients in the Mediterranean diet," Food Chem 70:319-23.

26. Walker J.R.L. Antimicrobial compounds in food plants. Pages 181204 in Dillon VM, Board RG, eds. Natural Antimicrobial Systems and Food Preservation. Wallingford UK: CAB International.1994.

How to cite this article: Muthukumaran $\mathrm{P}$, Kumaravel $\mathrm{S}$, Thomas N. Phytochemical, GC-MS and FT-IR Analysis of Papaver somniferum L. J Pharm Biolog Sci 2019;7(1):1-8. 